\title{
Editorial: standard neuropsychological assessments in neurosurgery
}

\author{
Hysse Birgitte Forchhammer $^{1}$ - Jannick Brennum ${ }^{2}$ (D)
}

Received: 8 June 2015 / Accepted: 12 June 2015 / Published online: 1 July 2015

(C) Springer-Verlag Wien 2015

In this issue of Acta Neurochirurgica, Zweifel-Zehnder et al. [2] recommend routine screening of SAH patients with a standardized set of neuropsychological assessments. Their project is highly applaudable and represents at the same time a substantial challenge. Neurosurgeons pay keen attention to neurological deficits easily recognized by standard neurological examination. We recognize prominent symptoms such as paresis, visual field deficits, sensory disturbances, and marked cognitive deficits, i.e., severe aphasia, memory disturbances, and overt executive function disturbances. However, mild and moderate cognitive deficits and behavioral changes are easily overlooked. Often these symptoms do not present themselves on the ward where there are only restricted demands on the patient's cognition and active participation in daily activities. Failure to identify cognitive deficits is likely to have dire consequences for the patient and their relatives. Families may break up, and patients may lose their jobs. These consequences could be avoided if the cognitive dysfunctions are identified and relevant rehabilitation is initialized. Perhaps the most important issue is that the patient, the family, and the employers are supported in adapting to the new situation.

Jannick Brennum

jannick.brennum@regionh.dk

1 Department of Neurology, Neuroscience Centre, Rigshospitalet - Glostrup Hospital, University of Copenhagen, Copenhagen, Denmark

2 Copenhagen Neurosurgery, Neuroscience Centre, Rigshospitalet, University of Copenhagen, Blegdamsvej 9,

2100 Copenhagen, Denmark
We often refer to pertinent early identification of cognitive deficits as "mind the gap"- the gap between the neurosurgical standard of being "neurosurgical intact" and standard of performance needed to be reintegrated in normal life.

The ideal solution would be an easily administered standardized neuropsychological screening tool with a high degree of sensitivity, specificity, and reproducibility. Such a tool does not exist. In the field of neuropsychology, the development and evaluation of standardized neuropsychological screening tests has a long history. However, the strive for development of such tools seems to have gained renewed and interdisciplinary interest. Healthcare professionals, political and patient organizations articulate the need for ways to identify patients in need of support and rehabilitation in an era with explicit focus on limited resources.

In Denmark, a recent [1] Danish Medical Technology Assessment (MTA) of neuro-rehabilitation from 2011 evaluated the need for standardized assessment tools. The MTA concluded that such instruments are needed, even though the scientific evidence for recommendation of standard procedures and tests were limited.

To sum up, there are a number of issues that need to be considered when developing, testing, and implementing neuropsychological screening programs.

\section{Ecological validity}

Neuropsychological tests providing valid and reliable identification of cognitive deficits are accessible. However, there is still a need for knowledge about their ability to predict ecological validity, i.e., to predict whether the patient will be able to resume their normal everyday life as they knew it before the disease, including social and work relations. 


\section{Complex brain and social systems call for complex measurement techniques}

We do not doubt that neuropsychological symptoms result in reduced quality of life and psychological challenges for patients and relatives. Neuropsychological deficits are, however, not the only parameters that determine outcomes measures such as "return to work". Factors not related to brain damage, such as socioeconomic, cultural, and structural factors play have variable influences. The variable influence of these factors may negate comparisons of standardized neuropsychological tests and there prognostic value between different parts of the world and even between different regions. For this very reason, the Danish MTA recommend a wider evaluation of the individual patients quality of life, function of everyday life, and participation in societal functions. Furthermore, it was recommend not to rely solely on neuropsychological evaluation but also to incorporate evaluations by speech-, ergo-, and physiotherapists.

\section{Time to test}

Our knowledge of how neuropsychological symptoms develop, progress or remise, are limited. More knowledge is also needed as to the most adequate time for testing and retesting in relation to the brain injury.

\section{Confounding factors}

Fatigue and depression are commonly encountered after $\mathrm{SAH}$ and other brain insults/diseases. The influence of the symptoms on the neuropsychological assessments and on the effectiveness of neuro-rehabilitation is still very unclear.

Zweifwl-Zehnder et al. 2015 have a grand aim, "to establish a nationwide uniform, standardized, and validated neuropsychological assessment battery that is optimally suited to assess the most prevalent deficits in aSAH patients". The setting is Switzerland where treatment of SAH is centralized to eight centers, that all participate in this effort. The authors have taken up the challenge very systematically. They are at the very beginning of an exciting scientific journey, where they have agreed upon a common protocol. However, the implementation of this protocol and the reaping and interpretation of data still lies ahead.

We find it premature to recommend this protocol, or any other protocol, as a standard for assessment of SAH patients before a clinical and scientific value has been demonstrated. The Swiss group is to be admired for the tremendous task they have taken upon themselves. It may be of great value for us all, our patients, and their relatives. The Swiss group does have the option to address many of the challenges outlined above in the years to come. We are looking forward to following the results and sincerely hope their protocol stands the test of time, not just in identifying cognitive deficits but also as a tool to identify patients that will benefit from rehabilitation.

\section{Conflicts of interest None.}

Funding No funding has been received for this study.

\section{References}

1. Sundhedsstyrelsen (2011) Sundhedsdokumentation. Hjerneskaderehabilitering-en medicinsk teknologivurdering. København: Sundhedsstyrelsen, Sundhedsdokumentation. Serienavn 2011 13(1)

2. Zweifel-Zehnder AE, Stienen MN, Chicherio C, Studerus-Germann A, Stefan Bläsi S, Rossi S, Gutbrod K, Schmid N, Beaud,V, Mondadori C, Brugger P, Sacco L, Müri R, Hildebrandt G, Fournier J-Y, Keller E, Regli L, Fandino J, Mariani L, Raabe A, Daniel RT, Reinert M, Robert T, Schatlo B, Bijlenga P, Schaller K, Monsch AU (2015) Call for uniform neuropsychological assessment after aneurysmal subarachnoid haemorrhage: Swiss Recommendations. Acta Neurochir 